\title{
EPIDEMIA DE DROGAS PSIQUIÁTRICAS: TIPOLOGIAS DE USO NA SOCIEDADE DO CANSAÇO
}

\author{
EPIDEMIA DE DROGAS PSIQUIÁTRICAS: \\ TIPOLOGÍAS DE USO EN LA SOCIEDAD DEL CANSANCIO \\ EPIDEMIC OF PSTCHIATRIC DRUG: \\ TTPOLOGIES OF USE IN THE BURNOUT SOCIETY
}

\author{
Rodrigo Alvarenga ${ }^{1}$ e Marcelo Kimati Dias ${ }^{2}$
}

${ }^{1}$ Pontifícia Universidade Católica do Paraná, Curitiba/PR, Brasil

${ }^{2}$ Universidade Federal do Paraná, Curitiba/PR, Brasil

\begin{abstract}
RESUMO: Este artigo tem por objetivo analisar o fenômeno da epidemia de uso de drogas psiquiátricas a partir da perspectiva do consumo cada vez mais disseminado e sua relação com a criação de subjetividades sociais que se constituem pela medicalização. Foram considerados dois diferentes contextos de uso que não necessariamente estão vinculados a diagnósticos. Em uma extremidade do fenômeno, identifica-se o consumo voltado para melhoria de desempenho em atividades competitivas de mercado, utilizando drogas como metilfenidato com a perspectiva de ultrapassar o desenvolvimento usual das práticas sociais e produtivas. Em outra extremidade, populações vulnerabilizadas que utilizam os psicotrópicos com o intuito de suportar experiências cotidianas de sofrimento que seriam intoleráveis de outra forma. A análise, apresentada na forma de ensaio, indicou um processo de uso acentuado de drogas psiquiátricas relacionado aos ideais de produtividade e desempenho ditados pelo padrão normativo da subjetividade neoliberal, o qual atinge particularmente as mulheres.
\end{abstract}

PALAVRAS-CHAVE: Drogas; Medicalização; Sociedade do cansaço.

RESUMEN: Este artículo tiene como objetivo analizar el fenómeno de la epidemia del consumo de drogas psiquiátricas desde la perspectiva de un consumo cada vez más extendido y su relación con la creación de subjetividades sociales que están constituidas por la medicalización. Se consideraron dos contextos de uso diferentes, que no están necesariamente vinculados a los diagnósticos. En un extremo del fenómeno, se identifica el consumo orientado a mejorar el desempeño en actividades competitivas de mercado, utilizando drogas como el metilfenidato con la perspectiva de ir más allá del desarrollo habitual de prácticas sociales y productivas. En el otro extremo, poblaciones vulnerables que consumen psicofármacos para sustentar las vivencias cotidianas de sufrimiento que de otro modo serían intolerables. El análisis, presentado en forma de ensayo, indicó un proceso de uso acentuado de drogas psiquiátricas relacionado con los ideales de productividad y desempeño dictados por el patrón normativo de la subjetividad neoliberal, que afecta particularmente a las mujeres.

PALABRAS CLAVE: Drogas; Medicalización; Sociedad del cansancio.

ABSTRACT: This article aims to analyze the phenomenon of the epidemic of psychiatric drug use from the perspective of increasingly widespread consumption and its relationship with the creation of social subjectivities that are constituted by medicalization. Two different contexts of use which are not necessarily linked to diagnoses were considered. At one end of the phenomenon, consumption aimed at improving performance in competitive market activities is identified, with drugs such as methylphenidate being used with the perspective of going beyond the usual development of social and productive practices. At the other end, there are vulnerable populations that use psychotropic drugs in order to support everyday experiences of suffering that would otherwise be intolerable. The analysis, presented in the form of an essay, indicated a process of accentuated use of psychiatric drugs related to the ideals of productivity and performance dictated by the normative pattern of neoliberal subjectivity, which particularly affects women.

KEYWORDS: Drugs; Medicalization; Burnout society. 


\section{Introdução}

O modelo atual da psiquiatria encontra-se colocado à disposição da sociedade de consumo e da cultura de mercado, bem como de seus efeitos sobre a saúde mental da população. Por consequência, a própria medicação se transforma em objeto de consumo atendendo a dois aspectos ambíguos e simultâneos. Ao mesmo tempo em que o modelo proporciona uma condição artificial em que se torna possível suportar a carga de cobrança sobre suas vidas, também permite ao sujeito contemporâneo ampliar suas capacidades laborais, acadêmicas e estéticas, ao menos no curso prazo.

O aumento do consumo de medicamentos psiquiátricos, especialmente antidepressivos, nos últimos 30 anos é alvo de preocupações da comunidade científica por diversos motivos. Whitaker (2017) faz uma cuidadosa discussão sobre a produção científica que deu fundamentação à medicalização social e mostra como os estudos que embasaram o modelo foram sendo contraditados por estudos posteriores, porém, as evidências com relação às fragilidades científicas associadas ao modelo não impediram sua gigantesca expansão.

Whitaker (2017) revela o conjunto de premissas equivocadas que se tornam uma narrativa popular sobre doenças psiquiátricas e seu tratamento. O modelo explicativo baseado exclusivamente no desequilíbrio neurofisiológico, ao qual os transtornos mentais estariam associados, não se sustenta como modelo etiológico ou como construção epistemológica. A medicação como forma de equilibrar o funcionamento dos neurotransmissores e as doses de serotonina e dopamina no cérebro, que estariam alteradas na esquizofrenia ou na depressão, por exemplo, não possui evidência científica.

Nesse sentido, estaríamos diante de uma epidemia das drogas psiquiátricas que medicaliza cada vez mais comportamentos cotidianos e restringe a noção de saúde mental a padrões de normalidade inalcançáveis, com base em uma base pseudocientífica. Tal epidemia tem alcances que não se restringem ao uso terapêutico como uma forma de lidar com sintomas, mas se estendem pelo mundo do trabalho, caracterizado pelo excessivo foco na performance profissional. Busca-se, também por meio das drogas psiquiátricas, uma espécie de melhoramento cognitivo voltado para o aumento da produtividade, desconsiderando as implicações éticas desse processo.

O uso de medicamentos ocorre em um contexto no qual o consumo expressa a necessidade de resposta a demandas pessoais, acadêmicas e profissionais na busca pela alta performance, ao mesmo tempo em que se acobertam os sofrimentos infligidos por essa lógica (Carneiro et al., 2013). Trata-se de um processo que aponta o lucro e o consumo como objetivo final da sociedade, do mesmo modo que promove o aumento progressivo da desigualdade social e da concentração de renda. Na sociedade neoliberal o uso de drogas lícitas ganha diferentes significados, acontece em diferentes contextos e adquire inúmeras funções.

Ao fomentar maior produtividade e rendimento da população, se eleva a demanda por drogas de desempenho, ao mesmo tempo que antidepressivos servem como recursos para lidar com as frustrações decorrentes desse modelo econômico que pressiona os sujeitos pela lógica do consumo. A droga passa, nesse sentido, a desempenhar um duplo papel: não apenas auxilia nos processos de busca de melhores resultados e maior performance em todas as esferas da vida, mas também se torna o meio necessário para lidar com o vazio de sentido que decorre da busca frenética pelo desempenho. 
Neste artigo, portanto, o objetivo é analisar o contexto da medicalização social pelo qual os sujeitos são pressionados a melhorar seus resultados e a lidar com as angústias e sofrimentos envolvidos nesse processo, por meio da medicalização, especialmente no caso de mulheres que acabam por acumular funções e realizar dupla ou tripla jornada. Desse modo, se visa a compreender os modos de subjetivação associados à epidemia das drogas psiquiátricas na sociedade atual, a partir de duas tipologias de uso: para o aumento da performance e do rendimento e para suportar o esgotamento físico e mental gerado por essa lógica. Para analisar essa polissemia das drogas psiquiátricas no mundo contemporâneo, será observada a relação entre medicalização, sociedade de consumo e sociedade do cansaço, tendo por base o papel central desempenhado pela economia de mercado na produção de uma cultura de excessos e na criação do papel social marcado pela lógica do consumo e do desempenho.

O estudo realizado foi de natureza bibliográfica e é apresentado na forma de ensaio, com destaque para alguns dados apresentados em outras pesquisas sobre os impactos das demandas produtivas no uso de medicamentos por jovens universitários e sobre a saúde mental e medicalização das mulheres. Ao final, foi possível perceber que, embora o processo de medicalização social atinja todas as pessoas, existe uma tendência maior de uso entre as mulheres, as quais são particularmente atingidas pelos parâmetros de produtividade e pelo esgotamento relacionado à cultura do desempenho, marcadas pela perspectiva de uma sociedade do consumo e do cansaço. Isso não porque exista alguma vulnerabilidade natural relacionada ao gênero, mas principalmente porque a desigualdade perpetrada pelo machismo impõe à mulher a necessidade de ter que se dedicar mais que os homens para terem papel de destaque no mundo acadêmico ou profissional, ao mesmo tempo em que as pressiona à condição de multitarefas, levando ao esgotamento psíquico.

\section{Medicalização e consumo}

Inicialmente torna-se importante salientar que a medicalização é um processo complexo que está relacionado não apenas à dispensa de medicamentos, mas também ao modo como a medicina passa a exercer controle sobre os mais diferentes aspectos da vida, tais como a moral, a sexualidade, higiene, hábitos alimentares, entre outros. Trata-se de uma espécie de ortopedia social relacionada diretamente ao modo que se exerce o poder sobre a população, sem que seja necessário atuar de forma coercitiva e repressiva, visto que o discurso médico assume um valor de verdade de caráter regulatório que é assumido pelos sujeitos. Conforme analisa Foucault (1984), com o capitalismo não se tem a instauração de uma medicina privada, mas uma coletivização da medicina pela socialização do corpo como objeto e força de trabalho.

O controle da sociedade sobre os indivíduos não se opera simplesmente pela consciência ou pela ideologia, mas começa no corpo, com o corpo. Foi no biológico, no somático, no corporal que, antes de tudo, investiu a sociedade capitalista. O corpo é uma realidade bio-política. A medicina é uma estratégia bio-politica. (Foucault, 1984, p. 47) 
A esse aspecto da medicalização relacionado à formação da medicina social e ao controle sobre os corpos por meio da sanitarização das cidades soma-se uma outra perspectiva encontrada no próprio Foucault, a saber, da medicalização enquanto um processo indefinido (Zorzanelli, Ortega, \& Bezerra, 2014). Indefinido, portanto, porque não se restringe aos elementos disciplinares que incidem sobre os corpos a partir de uma concepção sanitarista, mas extrapola os limites da própria medicina e incide sobre o desenvolvimento da sociedade como um todo. Segundo Zorzanelli e Cruz (2018, p. 724), “o processo de medicalização continua se referindo a uma intervenção médica, muitas vezes, autoritária. Entretanto, não mais relacionada apenas ao nível da política, mas a todo e qualquer aspecto da vida humana."

De acordo com Peter Conrad (1992), o processo de medicalização é descrito a partir de diferentes aspectos, tais como a identificação de um problema como uma disfunção médica, a criação uma terminologia para sua descrição e a criação de mecanismos de controle ou prescrição para intervenção do problema. Nesse processo, tornam-se médicos eventos cotidianos, compreendidos até então a partir de outros referenciais da cultura. É nessa concepção mais contemporânea de medicalização que se pauta a análise deste artigo, considerando o modo como o uso de psicotrópicos incide sobre a vida na sociedade capitalista neoliberal. Trata-se de uma perspectiva construcionista que enfatiza "o modo como o significado e a experiência da doença são moldados pelos sistemas sociais e culturais" (Conrad \& Barker, 2013, p. 187).

$\mathrm{O}$ aumento do consumo de medicações psiquiátricas tem forte impacto no mercado e, independente do papel que este fenômeno desempenha socialmente, o consumo de drogas psicotrópicas sustenta parte da indústria farmacêutica na atualidade. Dados levantados ao longo dos últimos 20 anos mostram que este segmento econômico se expande há décadas e que a venda de psicotrópicos tem cada vez maior importância relativa nesse mercado, ocupando até $23 \%$ do capital empregado por toda a indústria (Santos, 2014). O fenômeno de expansão do consumo de psicotrópicos é acompanhado por diferentes questões relacionadas ao seu significado. Esse fenômeno pode remeter à necessidade de explicações e o primeiro elemento a ser questionado é se o aumento do uso de psicotrópicos decorre exclusivamente do aumento da prevalência de doenças mentais.

A relação entre o aumento da prevalência de doenças e a ampliação do consumo é complexa e envolve outros elementos que impossibilitam que essa relação seja feita de forma direta. Com isso, caso a epidemia do uso de psicotrópicos correspondesse a uma epidemia de transtornos mentais, estaríamos diante de um momento histórico no qual o sofrimento mental estaria presente de forma inédita. Podemos listar a princípio dois aspectos dessa relação que impossibilitam a relação direta entre aumento de prevalência de sofrimento mental e aumento de consumo de drogas psicotrópicas. O primeiro ponto é que o desenvolvimento dos manuais diagnósticos envolve a resposta que fenômenos psíquicos têm ao uso de psicotrópicos. Um exemplo foi a criação do diagnóstico de transtorno de pânico, cuja categoria não envolve apenas a descrição de crises de ansiedade paroxística, uma vez que o desenvolvimento do diagnóstico envolveu estudos que evidenciavam respostas para os quadros relacionados com o uso de antidepressivos em baixas doses.

Em segundo lugar, o fenômeno de consumo de drogas psiquiátricas tem relação com as novas indicações de prescrição, que surgem conforme o conceito de doença mental se transforma a cada manual diagnóstico, estabelecendo novos parâmetros diagnósticos. 
Isto implica mudanças na epidemiologia das categorias psiquiátricas. Um exemplo é o esperado aumento de prevalência para transtornos depressivos em decorrência da diminuição do tempo mínimo de sintomas, ocorrido entre o DSM IV (American Psychiatric Association, 2002) e DSM-V (American Psychiatric Association, 2014), publicações elaboradas periodicamente pela Associação de Psiquiatria Norte-americana. Como exemplo das mudanças realizadas entre manuais diagnósticos, Araújo e Lotufo (2014, p. 74) apontam: "foi a retirada do luto como critério de exclusão do Transtorno Depressivo Maior. No DSM-5, é possível aplicar esse diagnóstico mesmo àqueles que passaram pela perda de um ente querido há menos de dois anos”. Uma vez que os critérios diagnósticos mudam, o mesmo ocorre com a indicação de uso de medicações antidepressivas. Portanto, a expansão do uso não pode ser entendida como referência para o aumento de prevalência de diagnósticos psiquiátricos, pois os parâmetros foram alterados periodicamente.

Além do aumento artificial da prevalência em função da mudança dos parâmetros diagnósticos, a elevação no índice de uso de drogas psiquiátricas tem relação com sua inserção no mercado. Nesse sentido, o medicamento, mesmo com uso mediante prescrição e condicionado a uma "necessidade", é um objeto de consumo e, como tal, integra o universo de "elementos da cultura material" que participam da constituição e afirmação de identidades e estilos de vida (Barbosa, 2009). E, nesse aspecto, é importante realizar uma discussão sobre como se estabelece a noção de necessidade de um produto a ser consumido. Por um lado, admite-se que a atividade de consumo busca satisfazer necessidades individuais; por outro, o consumo pode ser entendido como uma atividade que satisfaz necessidades de um sistema (e não de indivíduos). As necessidades individuais seriam criadas e moldadas culturalmente de modo a manter esse sistema em funcionamento. Dessa forma, compreender o medicamento como um produto de consumo e o seu lugar nesse sistema, assim como todos os atores envolvidos, pode apontar alguns caminhos no entendimento de importantes aspectos dessa "epidemia".

As indústrias farmacêuticas apresentam continuamente aumento de investimentos na área de marketing, atuando com os consumidores das drogas e junto aos prescritores (Barros, 1983; Silva, 2016). A abordagem da sociologia frente ao consumo pode ser dividida em diferentes perspectivas, sendo algumas mais ou menos predominantes em alguns períodos históricos. Uma delas enfatiza o papel da estrutura social nas determinações do objeto a ser consumido. A última perspectiva aborda especialmente os significados e atribuições simbólicas associadas às escolhas de consumo (Lipovetsky, 2007). O referido modelo considera particularmente forças sociais que condicionam o consumo a um processo de massificação e alienação.

Em uma outra perspectiva identificada como pós-moderna, autores como Bauman (2001) e Slater (2002) identificam o processo de consumir como uma forma de afirmar-se individualmente, com resgate do direito de escolha, a partir de um mecanismo que torna possível a criação de identidade em uma sociedade massificada. A discussão sobre a epidemia de uso de psicotrópicos se enriquece mediante um entendimento crítico do uso medicamentos em torno de seu consumo envolvendo questões como alienação, commoditização e dominação (Bradshaw \& Firat, 2007).

Partindo da noção de consumo como fenômeno social (e não individual ou grupal), é possível compreender como as esferas de produção e consumo são articuladas por meio do modelo clássico de consumo, que se baseia na premissa de que as necessidades são inatas, não podendo, portanto, ser manipuladas. Nesse sentido, o mercado teria a função 
de identificá-las e, então, criar alternativas para satisfazê-las, um pensamento que relega o consumo a um papel subordinado à produção. Segundo esse modelo tradicional, ainda que as necessidades sejam inatas, os desejos são culturalmente moldados. Entretanto, admite-se que essa distinção entre desejo culturalmente construído e necessidade tem limites imprecisos, na medida em que se torna cada vez mais difícil identificar até que ponto o indivíduo age movido por necessidades inatas ou estímulos da cultura.

Um segundo modelo procura evitar esta dicotomia entre desejo e necessidade, assim como é voltado para o processo de ressignificação do consumo. Nessa perspectiva, mesmo que o consumo seja um ponto fundamental na reprodução do capital, sua necessária articulação com a produção não é automática (Slater, 2002). Na medida em que as condições de produção mudam tanto quantitativamente (massificação) quanto qualitativamente (inovação), o mesmo ocorre na esfera do consumo: não só há uma expansão quantitativa como uma transformação das necessidades e a criação de novos valores de uso. Tais transformações, por sua vez, não advêm de necessidades humanas inatas, mas das necessidades de acumulação do sistema.

A estabilização de um regime de acumulação requer, portanto, um modo de regulação que não se restrinja à economia, mas a um conjunto de estruturas econômicas, culturais, políticas e, no caso, ficando a cargo de uma regulação de toda a sociedade capitalista. Para manter o sistema em funcionamento é necessário cada vez mais enriquecer tanto os valores de uso das mercadorias quanto as necessidades dos indivíduos, que são culturalmente criadas, bem como correspondem à demanda efetiva do mercado (Slater, 2002).

O significado social de um bem advém de sua condição de commodity, ou seja, o fato de ser um produto mercantilizado, produzido para a troca, no intuito de gerar lucro. Dessa forma, o processo de commoditização passa pelo enriquecimento de valor de uso e pela síntese de necessidades. Uma vez que a satisfação de necessidades depende cada vez mais de commodities, o mercado passa a figurar como uma instituição poderosíssima na sociedade contemporânea (Bradshaw \& Firat, 2007).

O elo entre produção e consumo para a satisfação de necessidades, antes sob o controle dos indivíduos, que produziam boa parte dos bens que precisavam, foi gradualmente perdido, sendo essa articulação quase que completamente mediada pelo mercado na sociedade ocidental. O poder do mercado não se concentra apenas na esfera econômica, mas na sociedade de uma forma sistêmica, sendo capaz não só de desenvolver quanto de modificar padrões de consumo segundo as demandas do sistema capitalista.

A sociedade atual, como descrita por Lipovetsky (2007), se caracteriza pelo hiperconsumo, a partir do qual se vislumbra felicidade e realização pessoal por meio do aumento da capacidade individual de compra. Em A felicidade paradoxal: ensaio sobre a sociedade do hiperconsumo, o autor analisa historicamente as mudanças nas relações de consumo, considerando que nas últimas décadas a perspectiva do mercado tomou conta de todos os espaços da vida humana, alterando profundamente a vivência do tempo.

Nessa perspectiva, todo o foco das práticas sociais está voltado ao tempo presente, comprometendo profundamente a conexão com o passado e a possibilidade de elaboração de um futuro. Tudo gira em torno da produtividade e do direito ao gozo, isto é, quanto mais os indivíduos são submetidos ao trabalho, entendem mais o direito ao prazer e ao relaxamento (que, por sua vez, é traduzido como consumo). 
A vida no presente sobrepôs-se às expectativas do futuro histórico, e o hedonismo, às militâncias políticas; a febre do conforto ocupou o lugar das paixões nacionalistas e os lazeres substituíram a revolução. Apoiando-se na nova religião da melhoria contínua das condições de vida, o melhor-viver tornou-se uma paixão das massas, o objetivo supremo das sociedades democráticas, um ideal exaltado em cada esquina. (Lipovetski, 2007, p. 07)

O referido modelo de sociedade focado no presente, que apresenta uma intensa demanda de prazer em uma cultura de excessos como busca da felicidade tem forte adequação ao consumo amplo de psicotrópicos. Essa relação não se dá exclusivamente a partir da hipótese de que esse modelo de sociedade implica sofrimento mental e na necessidade de se lidar com ele quimicamente, mas faz parte da forma como se constitui a produção da subjetividade na contemporaneidade.

\section{Consumo de psicotrópicos, produtividade e estratégia de desempenho}

Uma das características do desenvolvimento de psicotrópicos ao longo dos últimos 50 anos é a progressiva segurança e diminuição da toxicidade relacionada ao seu uso. Um dos exemplos é a substituição de barbitúricos por benzodiazepínicos a partir da década de 1960 (Huf, Lopes, \& Rozenfeld,_2000), mas, embora estes últimos fossem mais seguros que os primeiros, sua utilização pouco criteriosa implicou o surgimento de milhões de dependentes químicos (Tancredi \& Spinola, 1986). Da mesma forma, um dos principais motivos da explosão do uso de antidepressivos na década de 1990 ocorreu por meio da suposta segurança de sua utilização em comparação às medicações disponíveis no mercado até então (Brambilla, Cipriani, \& Hotopf, 2005). A suposta segurança na administração de psicotrópicos vem, ao longo desse processo, permitindo critérios progressivamente mais flexíveis para a prescrição dessas drogas. Ainda que, historicamente, processo muito semelhante tenha ocorrido, reportado e discutido há mais de 30 anos com o surgimento dos benzodiazepínicos, a expansão de usuários crônicos de antidepressivos mostra um fenômeno de consumo semelhante, com consequências ainda pouco previsíveis.

Dessa forma, a suposta segurança de psicotrópicos não é um fenômeno novo, is to é evidenciado pelos milhares de dependentes de benzodiazepínicos, mesmo que a dependência de tal classe de medicamentos seja descrita há muito tempo na literatura científica. $\mathrm{O}$ avanço da quantidade, eficácia e segurança no uso de psicotrópicos envolve fenômenos distintos. A expansão dessa forma específica de consumo é ainda facilitada por diferentes motivos. Entre eles: (a) a existência de um modelo de classificação da psiquiatria que é pautado na relação entre sintomas e medicamentos utilizados; (b) a consolidação de prescrições não baseadas em diagnósticos médicos, mas que obedecem a uma conveniência social e que remete diretamente aos fenômenos de identidade por intermédio do consumo, da tolerância e desempenho em situações cotidianas, ainda que a prescrição siga o procedimento de identificação de sintomas e atuação sobre eles. Neste segundo caso, a prescrição obedece a uma lógica médica, lança mão de sintomas e, principalmente, de um modelo neurobiológico que justificaria sua prescrição e uso. 
O melhor exemplo acerca da segunda situação é a expansão do uso de metilfenidato, especialmente em grupos sociais competitivos, como alunos de medicina. Uma pesquisa (Morgan et al., 2017) realizada com 200 estudantes de medicina demonstrou que 57,5\% deles já havia usado alguma substância psicoestimulante, tais como, energéticos, cafeína, ecstasy, piracetan e modafinil, sendo que 51,3\% iniciaram o uso durante o curso. As respostas mais comuns sobre os motivos do uso foram para compensar as horas de sono perdidas e melhorar o raciocínio, atenção ou memória. Considerando apenas o uso do metilfenidato, substância comercializada principalmente com o nome de Ritalina, a prevalência de consumo durante a vida chegou a 20\%, uma vez que 64\% iniciaram o consumo durante o curso de Medicina (Morgan et al., 2017). Um outro estudo apontou que entre $23,72 \%$ dos estudantes de medicina que faziam uso do metilfenidato, apenas 2,56 deles utilizavam com base em prescrição médica para o tratamento do Transtorno de Déficit de Atenção e Hiperatividade (TDAH) (Carneiro et al., 2013). Um estudo realizado entre alunos universitários na África do Sul identificou que 11,3\% dos alunos haviam feito uso de metilfenidato nos doze meses anteriores ao estudo. Desses, apenas 27,3 \% tinham diagnóstico que justificasse seu uso e 45\% dos usuários consumiam antes de avaliações com finalidade de aumentar a capacidade de atenção, concentração e memória.

Finger, Silva e Falavigna (2013) identificaram, em revisão bibliográfica, a prevalência de uso de metilfenidato de até $16 \%$ entre estudantes de medicina, ainda com grande maioria dos usuários sem possuir diagnósticos que justificassem o referido uso. As razões atribuídas ao uso remetiam à melhora do desempenho no estudo e em avaliações. A prevalência mais alta, em relação a estudantes de outras áreas, está associada ao fato de que os cursos de medicina são frequentemente mais competitivos, além da maior facilidade de acesso, ainda que muitos tenham obtido a medicação no mercado ilegal.

Utiliza-se com frequência o termo uso recreativo de drogas que têm a finalidade de obter estados de consciência alterados, mitigação da dor ou experimentar sensações extraordinárias em um contexto de busca por prazer, contudo, o consumo de metilfenidato tem como finalidade a melhoria de desempenho em um ambiente competitivo. Existe uma perspectiva, com o consumo, não de normalizar um comportamento ou controle de um fenômeno psíquico patológico, mas de sobreposição à própria normalidade. Não há a finalidade de alcançar a normalidade, não há procura do prazer, não há um movimento contrário ao sistema, mas um aprofundamento em sua adequação a ele.

\section{Psicotrópicos como estratégia de tolerância ao cotidiano}

Por um lado, se há um número crescente de alunos universitários que utilizam metilfenidato para melhora do desempenho em um ambiente competitivo, no qual pequenas diferenças de performance podem significar superar futuros adversários no mercado de trabalho, por outro, há um número crescente de pessoas que utilizam psicotrópicos de forma contínua como estratégia para suportar o cotidiano. A utilização de anfetaminas como estratégia para o aumento da produtividade e competitividade é emblemática e situa o fenômeno historicamente. Entretanto, quantitativamente, é pouco significativa em relação ao consumo global na epidemia de uso de medicações psiquiátricas que se concentra no uso de antidepressivos e ansiolíticos. Uma maneira de realizar esta análise é nos aprofundarmos no estudo da forma com que populações mais expostas e vulneráveis desenvolvem usos excessivos de psicotrópicos. 
Há alguns anos, a literatura em psiquiatria identifica uma maior prevalência de transtornos de humor, incluindo episódios de depressão em mulheres, quando em comparação por gênero. Diversos estudos apontam nesta direção: em uma investigação multicêntrica, com amostra superior a mil participantes, Lima et al. (2008) identificaram em estudo populacional o sexo feminino como variável significativa para uso de psicotrópicos (18\% das entrevistadas vinham em uso de psicotrópicos contra 5\% dos do sexo masculino). Da mesma forma, Andrade, Viana e Silveira (2006) realizaram uma revisão tendo como referência grandes estudos populacionais desenvolvidos nos EUA, Inglaterra, Escócia, São Paulo e Santiago do Chile. Tal revisão apontou mulheres como mais sujeitas aos transtornos de humor e uso de medicamentos psicotrópicos. Em amostragens nacionais, identifica-se o mesmo fenômeno de prevalência feminina entre usuários de psicotrópicos. Um estudo populacional feito em Pelotas, no Rio Grande do Sul, com mais de 1200 entrevistados, encontrou, tal como no restante da literatura da área, diferenças significativas entre uso de psicotrópicos por mulheres (11,6\%) e homens (4,8\%) (Garcias et al., 2008).

Ainda em um contexto nacional, Moura et al._(2016) viabilizaram uma revisão sobre fatores associados ao uso de psicotrópicos de forma abusiva na Atenção Básica e encontraram os mesmos dados, isto é: medicamentos psiquiátricos são utilizados em elevadas doses e por mais tempo pelas mulheres, sendo que o gênero constitui um fator de risco para uso de psicotrópicos e desenvolvimento de dependência. Outros estudos nacionais corroboram a identificação do fenômeno. Vidal et al. (2013) revelaram uma prevalência de 30\% de uso de psicotrópicos em Barbacena, MG. Prado et al. (2017), em estudo populacional transversal em Campinas-SP, identificou o sexo feminino como fator de risco para utilização de medicações psicotrópicas.

O uso crônico de psicotrópicos por pacientes do sexo feminino, com tempo de uso e doses excessivamente altas, é uma realidade anterior ao surgimento de antidepressivos chamados inibidores de receptação de serotonina, especialmente na rede pública. Durante a década de 1990, um estudo feito no interior de São Paulo apontou que o uso de benzodiazepínicos na rede pública era maior e mais prolongado entre usuárias do sexo feminino e a média do tempo de uso era de 15 anos. Importante salientar que o tempo médio preconizado é de menos de 30 dias, uma vez que esses medicamentos desenvolvem facilmente dependência (Ribeiro et al., 2007).

Nordon et al. (2009) identificou em uma unidade básica de saúde no interior de São Paulo que, entre usuárias da atenção primária, a utilização de benzodiazepínicos chegava a $13,14 \%$ das entrevistadas. O uso era mais frequente entre mulheres analfabetas, com união estável e entre os 50 e 69 anos de idade. Os dados sugerem que, além do fato em si de ser mulher, alguns aspectos relacionados com elas favorecem o uso e desenvolvimento de dependência de psicotrópicos, como as condições de trabalho e a situação econômica.

Diferentes estudos (Lima et al., 2008; Moura et al., 2016) apontam que no Brasil a ocupação de dona de casa implica risco quanto ao uso de psicotrópicos, podendo chegar até 26,6 \% de prevalência em estudos populacionais (Lima et al., 2008). Araújo e Lotufo (2014) realizaram estudo transversal com mulheres em Feira de Santana, na Bahia, com a utilização de questionário autoaplicável voltado para a identificação de transtornos mentais comuns.

Os autores identificaram que, quanto maior for a sobrecarga doméstica, quanto menor for a ajuda e quanto menor for a renda, maior será o escore da avaliação, com mais prevalência de transtorno mental. O estudo identificou ainda que ser chefe de família, ter menor escolaridade, ser negra ou parda, além de pouco lazer figuram como fatores 
que estavam relacionados a esta maior prevalência. Algumas hipóteses são levantadas para explicar o fenômeno; a primeira é que donas de casa têm mais disponibilidade de tempo para acessar serviços públicos de saúde, e mais de um estudo citado mostrou que acesso aos serviços de saúde apresenta forte relação com uso de psicotrópicos.

Neste modelo de sociedade hedonista, consumista e competitivo, donas de casa aparecem com uma espécie de resquício de outros momentos do capitalismo teoricamente superados. Elas sofrem um processo de desvalorização social que explica a maior vulnerabilidade de desenvolvimento de dependência ou uso abusivo de psicotrópicos. As demais variáveis associadas com o sofrimento mental, isto é, cor de pele, renda, escolaridade e lazer apontam para uma forte associação entre sofrimento mental, baixa renda, discriminação e desqualificação social.

Ainda nesse sentido, a desvalorização de atividade profissional não é uma exclusividade da atividade de dona de casa. Prestes e Bezerra (2019) mostraram, por intermédio de pesquisa desenvolvida no Estado do Paraná, uma diferença salarial baseada em gênero na qual as mulheres têm salário inferior. A este fenômeno, que implica perda de poder financeiro, agrava-se a situação feminina pelo fato de que desenvolver atividade remunerada não exclui socialmente a mulher de suas atribuições de dona de casa, elevando sua vulnerabilidade social e psicológica, em face das demandas crescentes e baixo reconhecimento por suas atividades.

Desse modo, os ditames de uma sociedade de consumo pautada pela ideologia do desempenho como modo de realização pessoal e profissional onera, sobremaneira, as mulheres - desde aquilo que se refere aos apelos estéticos a padrões inalcançáveis de beleza e perfeição, como também pela necessidade de corresponder ao mercado competitivo, sem faltar com as obrigações que se constituíram historicamente como cuidar dos filhos, fazer as refeições, lavar e organizar as roupas e realizar a limpeza da casa. Ou seja, a mulher é a vítima primeira da sociedade do desempenho promovida pelo sistema político e econômico neoliberal que se impõe a toda a América Latina.

A busca incessante por produtividade e melhor rendimento das máquinas e das pessoas tem gerado uma sociedade na qual torna-se imperativo explorar-se a si mesmo como forma de realização, visto que se impõe em todos os âmbitos a necessidade de obter sucesso e ser perfeito. Os efeitos sociais e psicológicos desse padrão de sociedade, que cobra cada vez mais e melhores resultados das pessoas, podem estar diretamente associados aos sintomas de ansiedade, estresse, depressão, entre outros. Nesse sentido, a sociedade do consumo, focada na ideologia do desempenho, estaria levando os indivíduos a entrarem em colapso pelo alto grau de exigência, fruto de uma sociedade do cansaço (Han, 2015).

Um dos elementos presentes dessa condição de estafa internalizada psiquicamente é o incentivo e cobrança da capacidade de fazer várias coisas ao mesmo tempo, um retorno a uma forma de se viver em que ser multitarefas é sinal de competência técnica e profissional, como se cada um fosse uma empresa de si mesmo. O processo de medicalização, portanto, estaria inserido dentro desse contexto, visto que o grau de frustração e esgotamento passam a exigir um modo de adaptação aos padrões de exigências que apenas se sustenta quimicamente.

O doping não passa de uma consequência dessa evolução na qual a própria vitalidade, que é constituída por um fenômeno bastante complexo, é reduzida a uma função vital e um desempenho vital. Como contraponto, a sociedade do desempenho e a sociedade ativa geram um cansaço e esgotamento excessivos... O excesso da elevação do desempenho leva a um infarto da alma. (Han, 2015, p. 70) 
Trata-se de uma sociedade que produz o próprio sintoma, visto que inflige à população um alto grau de sofrimento, pelo sacrifício de suas vidas em nome do alto rendimento, mas que encontra o meio químico pelo qual pode continuar operando, ao menos por mais alguns instantes. Tais características da sociedade do cansaço e da lógica do desempenho precisam ser consideradas a partir de uma perspectiva de gênero, visto que a desigualdade oriunda do machismo faz com que o homem tenha inúmeros privilégios quando comparada sua condição com a das mulheres. Além disso, se faz necessária considerar a perspectiva de classe social, visto que a dominação e a violência ocorrem de forma ainda mais acentuada contra mulheres de baixa renda, mestiças ou negras, o que se evidencia no próprio atendimento em saúde mental oferecido. Se em mulheres brancas a doença mental é concebida "como uma condição patológica que precisa ser estudada e explicada, nas outras seria concebida como decorrência inevitável das degenerescências raciais e/ou da inferioridade sociocultural” (Engel, 2008, p. 185).

A forma como se constitui a subjetividade feminina na sociedade capitalista é marcada pela inferiorização como estratégia de dominação. Ao se atribuir o papel de cuidadora e responsável pelos filhos e pela harmonia do lar, ao mesmo tempo em que se exige desempenho acadêmico e profissional, além de todos os apelos relacionados ao padrão estético e sexual, cria-se uma meta inalcançável que contribui para o sentimento de frustração e angústia, que pode comprometer a autoestima e a saúde mental. Desse modo, "as desigualdades sociais envolvem os principais sentimentos relacionados à depressão e a outros transtornos mentais, como humilhação, inferioridade, percepção de falta de controle sobre o meio e impotência” (Ludermir, 2008, p. 661). Uma das características da sociedade do cansaço é, justamente, a produção de uma subjetividade que se percebe sempre em dívida consigo mesma, nunca se é aquilo o que deveria ser. Trata-se de uma forma de opressão que atinge diretamente os sujeitos em sua intimidade psíquica, colocando-os em situação de vulnerabilidade frente aos transtornos mentais e à medicalização, seja como meio de se adequar aos padrões incentivados de desempenho, seja para lidar com os efeitos do grau excessivamente elevado de exigências que são internalizadas e tornam-se insuportáveis.

\section{Considerações finais}

Conforme foi possível observar pelos dados relacionados à medicalização no âmbito universitário, a sociedade do desempenho, que prioriza o lucro em detrimento da saúde mental, não encontra outra forma de se realizar senão pela potencialização química dos resultados, com a intenção deliberada de aumento da concentração e da capacidade produtiva. Os dados relativos ao consumo de medicamento por mulheres deixaram claro o modo como os ditames da cultura do al to rendimento e performance idealizada e inalcançável pode levar ao colapso.

Ignorando essa relação entre sistema produtivo e econômico, trabalho e esgotamento psíquico, a psiquiatria hegemônica e a lógica da medicalização se pautam pela compreensão de que os transtornos mentais são defeitos de natureza cerebral, que decorrem de desequilíbrios neurológicos e bioquímicos, que devem ser regulados por meio das drogas psiquiátricas. Assim, os sintomas associados ao sofrimento cujo modo de vida contemporâneo gera são reduzidos a determinados defeitos individuais, relacionados ao mal funcionamento da bioquímica cerebral, mas que podem ser resolvidos pela dispensa de medicamentos, como se para encobrir as falhas do sistema social. 
O modo como tal modelo de sociedade se organiza atinge principalmente mulheres, visto que a desigualdade de gênero as coloca em uma condição de dupla exploração. No trabalho enfrentam a exclusão velada e a necessidade de ser potencialmente mais produtivas que os homens para alcançar os mesmos cargos e promoções, normalmente com salários mais baixos para realizar as mesmas atividades. Em casa estão sujeitas ao mesmo tipo de subalternidade e acabam acumulando funções na criação dos filhos e em vários outros aspectos frente aos quais os homens não se percebem como exploradores privilegiados e, muitas vezes, nem as próprias mulheres se dão conta dessa condição, ou não têm força para lutar contra.

O esgotamento psíquico nesses casos, decorrente das pressões sociais impostas pelo sistema neoliberal, no qual o machismo é uma de suas facetas, vulnerabiliza especialmente o gênero feminino, daí o sentido dos índices mais elevados de transtornos psiquiátricos e uso de medicalização por parte das mulheres. Diante desse cenário, urge a necessidade de discutir os sintomas relacionados à sociedade do cansaço gerados pela competitividade produzida e estimulada pelo modelo econômico neoliberal, de modo a contrapor o naturalismo da biomedicina e a epidemia da medicalização como formas de tratamento ao sofrimento imposto pelo cotidiano da exploração da mão de obra e de um gênero sobre outro.

\section{Referências}

American Psychiatric Association. (2002). Manual diagnóstico e estatístico de transtornos mentais: DSM-4 (4. ed.). Artmed.

American Psychiatric Association. (2014). Manual diagnóstico e estatístico de transtornos mentais: DSM-5 (5 $5^{\mathrm{a}}$ ed.). Artmed.

Andrade, L. H. S. G., Viana, M. C., \& Silveira, C. M. (2006). Epidemiologia dos transtornos psiquiátricos na mulher. Archives of Clinical Psychiatry, 33(2), 43-54. https://dx.doi. org/10.1590/S0101-60832006000200003.

Araújo, Á. C. \& Lotufo N. F. (2014). A nova classificação americana para os transtornos mentais: o DSM-5. Revista Brasileira de Terapia Comportamental e Cognitiva, 16(1), 67-82. Recuperado de http://pepsic.bvsalud.org/scielo.php?script $=$ sci arttext\&pid $=\mathrm{S} 1517-55452014000100007 \& \mathrm{ln}$ $\mathrm{g}=\mathrm{pt} \& \mathrm{t} \operatorname{lng}=\mathrm{pt}$.

Barbosa, L. (2009). Sociedade de consumo (3 $3^{\mathrm{a}}$ ed.). Zahar.

Barros, J. A. C. (1983). Estratégias mercadológicas da indústria farmacêutica e o consumo de medicamentos. Revista de Saúde Pública, 17(5), 377-386. https://dx.doi.org/10.1590/So03489101983000500003.

Bauman, Z. (2001). Modernidade líquida. Zahar.

Bradshaw, A. \& Firat, A. F. (2007). Rethinking critical marketing. In M. Saren \& P. Svensson (Orgs.), Critical marketing: defining the field (pp. 30-40). Butterworth-Heinemann.

Brambilla, P., Cipriani, A., \& Hotopf, M. C. (2005). Side-Effect profile of fluoxetine in comparison with other SSRIs, tricyclic and newer antidepressants: a meta-analysis of clinical trial data. Pharmacopsychiatry, 38(2), 69-77. 
Carneiro, S. G., Prado A. S. T., Araujo, E. C. J., Moura, H. C., Strapasson, J. F., \& Rabelo, N. F. (2013). O uso não prescrito de metilfenidato entre acadêmicos de Medicina. CadUniFOA, 1, 53-59.

Conrad, P. \& Barker, K. (2013). A construção social da doença: insights-chave e implicações para políticas de saúde. Ideias, 2(2), 183-219. Recuperado de https://periodicos.sbu.unicamp. br/ojs/index.php/ideias/article/view/8649322.

Conrad, P. (1992). Medicalization and Social Control. Annual Review Of Sociology, 18, 209232. https://doi.org/10.1146/annurev.so.18.080192.001233.

Engel, M. G. (2008). Sexualidades interditadas: loucura e gênero masculino. História, Ciências, Saúde-Manguinhos, 15(Supl.), 173-190. https://doi.org/10.1590/So 104-59702008000500009

Foucault M. (1984). Microfisica do poder (Roberto Machado, org., rev. e introd.). Graal.

Finger, G., Silva, E. R., \& Falavigna, A. (2013). Use of methylphenidate among medical students: a systematic review. Revista da Associação Médica Brasileira, 59(3), 285-289. https:// dx.doi.org/10.1016/j.ramb.2012.10.007.

Garcias, C. M. M., Pinheiro, R.T., Garcias, G. L., Horta, B. L., \& Brum, C. B. (2008). Prevalência e fatores associados ao uso de antidepressivos em adultos de área urbana de Pelotas, Rio Grande do Sul, Brasil, em 2006. Cadernos de Saúde Pública, 24(7), 1565-1571. https://doi.org/10.1590/S0102-311X2008000700011.

Han, B-C. (2015). Sociedade do cansaço (E. Giachini, trad.). Vozes.

Huf, G., Lopes, C. S., \& Rozenfeld, S. (2000). O uso prolongado de benzodiazepínicos em mulheres de um centro de convivência para idosos. Cadernos de Saúde Pública, 16(2), 35 1-362. https://doi.org/10.1590/S0102-311X2000000200006.

Lima, M. C. P., Menezes, P. R., Carandina, L., Cesar, C. L. G., Barros, M. B. A., \& Goldbaum, M. (2008). Transtornos mentais comuns e uso de psicofármacos: impacto das condições socioeconômicas. Revista Saúde Pública, 42(4), 717-723. Recuperado de http://www.scielo.br/ scielo.php? script $=$ sci arttext\&pid $=$ S0034-89102008000400019\&lng $=$ en .

Lipovetsky, G. A. (2007). Felicidade paradoxal: ensaio sobre a sociedade do hiperconsumo. (M. Machado, trad.). Companhia das Letras.

Ludermir, A. B. (2008). Desigualdades de classe e gênero e saúde mental nas cidades. Physis: RevistadeSaúde Coletiva, 18(3), 451-467.https://dx.doi.org/10.1590/So103-73312008000300005

Morgan, H. L., Petry, A. F., Licks, P. A. K., Ballester, A. O., Teixeira, K. N., \& Dumith, S. C. (2017). Consumo de estimulantes cerebrais por estudantes de medicina de uma universidade do extremo sul do Brasil: prevalência, motivação e efeitos percebidos. Revista Brasileira de Educação Médica, 41(1), 102-109. https://dx.doi.org/10.1590/1981$\underline{-52712015 \mathrm{v} 41 \mathrm{n} 1 \mathrm{rb} 20160035 \text {. }}$

Moura, D. C. N., Pinto, J. R., Martins, P., Pedrosa, K. A., \& Carneiro, M. C. D. (2016). Uso abusivo de psicotrópicos pela demanda da estratégia saúde da família: revisão integrativa da literatura. Sanare, 15(2), 136-144. Recuperado de https://sanare.emnuvens.com.br/sanare/ article/view/1048.

Nordon, D. G., Alkamine, K., Novo, N. F, \& Hübner, C. K. (2009). Características do uso de benzodiazepínicos por mulheres que buscavam tratamento na atenção primária. Revista de Psiquiatria-RS, 31(3), 152-158. https://doi.org/10.1590/S0101-81082009000300004 
Prado, M. A. M. B., Francisco, P. M. S. B., \& Barros, M. B. A. (2017). Uso de medicamentos psicotrópicos em adultos e idosos residentes em Campinas, São Paulo: um estudo transversal de base populacional. Epidemiologia e Serviços de Saúde, 26(4), 747-758. https://doi.org/10.5123/ s1679-49742017000400007.

Prestes, A. F. \& Bezerra, F. M. (2019). Desigualdade salarial entre gêneros: análise por setor e região do Paraná. Economia E̊ Região, 7(2), 29-46. Recuperado de http://www.uel.br/revistas/uel/index.php/ecoreg/article/view/33644.

Ribeiro, C. S., Azevedo, R. C. S., Franco, V. S., \& Botega, N. J. (2007). Uso crônico de diazepam em unidades básicas de saúde: perfil de usuários e padrões de uso. São Paulo Medical Journal, 125(5), 270-274. https://doi.org/10.1590/S1516-31802007000500004.

Santos, D. V. D. (2014). A gestão autônoma da medicação: da prescrição à escuta. Tese de Doutorado em Saúde Coletiva, Universidade Estadual de Campinas, Campinas, São Paulo.

Silva, J. N. (2016). Epistemologia psiquiátrica e marketing farmacêutico: novos modos de subjetivação. Stylus, 33, 227-239. Recuperado de http://pepsic.bvsalud.org/scielo.php?script=sci arttext\&pid=S1676-157X2016000200018\&lng=pt\&tlng=pt.

Slater, D. (2002). Cultura do consumo e modernidade. Nobel.

Tancredi, F. B. \& Spinola, A. W. P. (1986). Consumo de medicamentos benzodiazepinicos no Brasil - 1970 a 1985: análise comparativa de tendências. Universidade de São Paulo. Recuperado de http://www.teses.usp.br/teses/disponiveis/6/6135/tde-26072016-172521.

Vidal, C. E. L., Yañez, B. F. P., Chaves, C. V. S., Yañez, C. F. P., Michalaros, I. A., \& Almeida, L. A. S. (2013). Transtornos mentais comuns e uso de psicofármacos em mulheres. Cadernos Saúde Coletiva, 21(4), 457-464. https://doi.org/10.1590/S1414-462X2013000400015.

Whitaker, R. (2017). Anatomia de uma epidemia: pílulas mágicas, drogas psiquiátricas e o aumento assombroso da doença mental. (V. Ribeiro, trad.). Editora Fio Cruz.

Zorzanelli, R. T., Ortega, F., \& Bezerra, B. (2014). Um panorama sobre as variações em torno do conceito de medicalização entre 1950-2010. Ciência É Saúde Coletiva, 19(6), 1859-1868. https://doi.org/10.1590/1413-81232014196.03612013.

Zorzanelli, Rafaela T. \& Cruz, Murilo G. Amancio. (2018). O conceito de medicalização em Michel Foucault na década de 1970. Interface - Comunicação, Saúde, Educação, 22(66), 72 1-731. https://doi.org/10.1590/1807-57622017.0194. 
RODRIGO ALVARENGA

https://orcid.org/0000-0001-8546-4442

Professor do Programa de Pós-graduação em direitos humanos e políticas públicas da Pontifícia Universidade Católica do Paraná. Doutor em Filosofia pela Universidade Federal de Santa Catarina com a realização de estágio de pesquisa na Universidade da Sorbonne em Paris.

E-mail: alvarenga.rodrigo@pucpr.br

\section{MARCELO KIMATI DIAS}

https://orcid.org/OOOO-0001-9190-8222

Doutor em Ciências Sociais pela Universidade Estadual de Campinas (2007)

e Mestre em Ciências Médicas pela Universidade Estadual de Campinas (2002). Professor de Saúde Coletiva da UFPR.

E-mail: mdkimati@gmail.com

\begin{tabular}{|c|c|}
\hline Histórico & $\begin{array}{l}\text { Submissão: 01/04/20 } \\
\text { Revisão: 14/07/2020 } \\
\text { Aceite: 31/07/2020 }\end{array}$ \\
\hline $\begin{array}{l}\text { Contribuição } \\
\text { dos autores }\end{array}$ & $\begin{array}{l}\text { Concepção: R.A.; M.K.D. } \\
\text { Coleta de dados: R.A.; M.K.D. } \\
\text { Análise de dados: R.A.; M.K.D. } \\
\text { Elaboração do manuscrito: R.A.; M.K.D. } \\
\text { Revisões críticas de conteúdo intelectual importante: } \\
\text { R.A.; M.K.D. } \\
\text { Aprovação final do manuscrito: R.A.; M.K.D. }\end{array}$ \\
\hline Financiamento & Não houve financiamento. \\
\hline
\end{tabular}

